\title{
Genomic and Genetic Studies in Autism Spectrum Disorders
}

\author{
Moyra Smith* \\ University of California, Irvine, CA, USA
}

Article Info

\section{Article Notes}

Received: June 28, 2016

Accepted: July 13, 2016

\section{*Correspondence:}

Moyra Smith,

University of California, Irvine, CA, USA

E-mail: dmsmith@uci.edu

C) 2016 Smith M. This article is distributed under the terms of the Creative Commons Attribution 4.0 International License.

\section{Keywords}

Canakinumab

$\mathrm{IL}-1 \AA$

autoinflammatory diseases

\section{Introduction}

The primary manifestations of autism spectrum disorders are deficits in social and emotional reciprocity, deficits in non-verbal communication; an additional manifestation is the occurrence of restrictive repetitive patterns of behavior ${ }^{1}$. Autism may occur as a one of the features present in particular syndromic disorders that involve additional defined clinical and pathological features, e.g. Tuberous Sclerosis, Rett syndrome etc. However, the majority of cases are nonsyndromic and autism is the key clinical manifestation.

In 2012 we reported results of analysis of genomic segmental copy number variants (CNVs) in 69 cases of autism including monozygotic twins and affected sib-pairs and on 35 parents of autistic individuals ${ }^{2}$. CNVs analyzed included regions that contained at least 20 markers. We analyzed the relative frequencies of CNVs in our study population relative to frequencies of CNVs in an available control data set (CEU). Results of our analyses revealed that the numbers of CNVs present were higher in the autism individuals than in their parents and higher than in controls. The number of CNVs present in parents was higher than the number of CNVs present in controls. In autistic individuals the predominant categories of genes present in CNVs greater than $1 \mathrm{MB}$ in size included genes with ion channel related functions and genes that had mitochondrial related functions.

The ion channel related genes included 6 genes that function at synapses GABRA5, GABRB3, GABRG3, CHRNA7, GPM6, and CACNA1C. The mitochondrial function genes included genes that encode subunits of electron transfer complexes, and genes that encode proteins that transport specific molecules across mitochondrial membranes. It is interesting to consider our report in the context of recent publications. Since 2012 comprehensive data have been generated relating to $\mathrm{CNV}$ analyses in autistic subjects and controls and in addition studies on exome sequencing on large numbers of cases of autism and controls have been published.

\section{CNV analyses}

In 2013 Moreno-De Luca et al. analyzed data from three different autism spectrum cohorts (SSC AGRE and AGP) ${ }^{3}$. Their analyses included 3955 individuals diagnosed with autism. From their analyses they identified recurrent deletions in the ASD cohorts at the following chromosome positions: 16p11.2, 15q13.215q13, 16p13.11, 16p12.1, 17q12, 1q21, 1q21.1, 3q29, 5q35, 16p11.2, 22q11.2. In some cases of autism duplications occurred at these chromosome positions.

Pinto et al. 2014 carried out copy number variant analyses on 2446 individuals with autism and their parents and they also assembled data on copy number variants present in 2640 unrelated control individuals ${ }^{4}$. The Illumina $1 \mathrm{M}$ arrays were used for CNV analyses. They reported that copy number variants were present in $4.7 \%$ of autism-affected individuals and in 1 to 2 percent of controls. The de novo CNVs found in autism-affected individuals were on average larger than those found in control individuals. The average length of CNVs in autism individuals was $1.17 \mathrm{Mb}$ while in control individuals the average length of CNVs was $0.55 \mathrm{MB}$. Importantly the number of genes impacted by CNVs was 3.8 fold higher in autism-affected individuals than in controls. In cases where the parental origin of CNVs could be determined, the numbers found to be of paternal origin where equal to the numbers found to be of maternal origin. Pinto et al. reported that $64 \%$ 
of the CNVs that were considered to be pathogenic were de novo in origin in the autistic individuals. Pinto et al. reported that brain expressed genes were more frequently impacted in the CNVs found in autistic individuals than in the CNVs found in control individuals; this was particularly the case in deletion CNVs.

\section{Exome sequencing studies}

In recent years a number of large scale sequencing studies on autistic individuals and their parents have revealed that rare de novo damaging loss of function mutations occurred in autism cases. Rubeis et al. (2014) carried out exome sequencing on 3,871 autism cases and 9,937 ancestry-matched or parental controls ${ }^{5}$. They identified de novo loss-of-function mutations in over $5 \%$ of autistic subjects. These mutations impacted 129 genes. These genes were reported to include voltage-gated ion channels histone-modifying enzymes and chromatin remodelers-particularly those involved in post-translational lysine methylation/demethylation modifications of histones. Additional gene sequencing studies have been carried out by several groups. It is important to note that defects in 200-1000 genes have been implicated in autism ${ }^{6}$.

\section{Autism risk genes identified through merged analyses}

The Simons Foundation autism research initiative SFARI (https://sfari.org/ merges information from multiple studies and compiles lists of genes implicated in autism including syndromic and non-syndromic autism. In addition, the genes listed have been implicated through analyses on copy number variants and through sequencing analyses. The SFARI gene list also includes the number of published reports that implicate a specific gene. Table 1 in this review lists 50 genes reported in SFARI as being involved in autism spectrum disorder in 15 or more publications.

\begin{tabular}{|l|c|l|}
\hline Gene symbol & $\begin{array}{c}\text { Number of } \\
\text { reports }\end{array}$ & Gene Names \\
\hline AHI1 & 17 & Abelson helper integration site 1 \\
\hline ANK3 & 18 & ankyrin 3, node of Ranvier \\
\hline $\begin{array}{l}\text { ANKRD4 } \\
\text { (PPP1R16B) }\end{array}$ & 17 & protein phosphatase 1 regulatory subunit 16B \\
\hline ARID1B & 17 & AT-rich interaction domain 1B \\
\hline AVPR1B & 16 & arginine vasopressin receptor 1B \\
\hline CACNA1C & 35 & calcium voltage-gated channel subunit alpha1 C \\
\hline CACNA1H & 19 & calcium voltage-gated channel subunit alpha1 \\
\hline CDKL5 & 22 & cyclin dependent kinase like 5 \\
\hline CHD8 & 18 & chromodomain helicase DNA binding protein 8 \\
\hline CNTN4 & 17 & contactin 4 \\
\hline CNTNAP2 & 49 & contactin associated protein-like 2 \\
\hline DMD & 33 & dystrophin \\
\hline DPP6 & 22 & dipeptidyl peptidase like 6 \\
\hline DYRK1A & 19 & $\begin{array}{l}\text { dual specificity tyrosine phosphorylation } \\
\text { regulated kinase }\end{array}$ \\
\hline EN2 & 18 & engrailed homeobox 2 \\
\hline FMR1 & 38 & fragile X mental retardation 1 \\
\hline FOXP2 & 28 & forkhead box P2 \\
\hline GABRB3 & 30 & $\begin{array}{l}\text { gamma-aminobutyric acid type A receptor } \\
\text { beta3 subunit }\end{array}$ \\
\hline GRIN2A & glutamate ionotropic receptor NMDA type \\
\hline
\end{tabular}

\begin{tabular}{|l|l|l|}
\hline GRIN2B & 25 & $\begin{array}{l}\text { glutamate ionotropic receptor NMDA type } \\
\text { subunit 2B }\end{array}$ \\
\hline HOXA1 & 15 & homeobox A1 \\
\hline IL1RAPL1 & 18 & interleukin 1 receptor accessory protein like 1 \\
\hline KCNMA1 & 16 & $\begin{array}{l}\text { potassium calcium-activated channel subfamily } \\
\text { M alpha 1 }\end{array}$ \\
\hline MACROD2 & 15 & MACRO domain containing 2 \\
\hline MBD5 & 22 & methyl-CpG binding domain protein 5 \\
\hline MECP2 & 50 & methyl-CpG binding protein 2 \\
\hline MET & 32 & MET proto-oncogene, receptor tyrosine kinase \\
\hline NF1 & 20 & Neurofibromin 1 \\
\hline NLGN3 & 30 & Neuroligin 3 \\
\hline NLGN4X & 30 & neuroligin 4, X-linked \\
\hline NLGN4Y & 26 & neuroligin 4, Y-linked \\
\hline NRXN1 & 61 & neurexin 1 \\
\hline OXTR & 34 & oxytocin receptor \\
\hline PTEN & 34 & phosphatase and tensin homolog \\
\hline RBFOX1 & 39 & RNA binding protein, fox-1 homolog 1 \\
\hline RELN & 28 & Reelin \\
\hline RORA & 15 & RAR related orphan receptor A \\
\hline SCN1A & 31 & sodium voltage-gated channel alpha subunit 1 \\
\hline SCN2A & 30 & sodium voltage-gated channel alpha subunit 2 \\
\hline SHANK2 & 19 & SH3 and multiple ankyrin repeat domains 2 [ \\
\hline SHANK3 & 49 & SH3 and multiple ankyrin repeat domains 3 \\
\hline SLC25A12 & 19 & $\begin{array}{l}\text { Solute carrier 25A12, (calcium-binding } \\
\text { mitochondrial carrier protein) }\end{array}$ \\
\hline SLC6A4 & 22 & $\begin{array}{l}\text { solute carrier family 6 member 4 (serotonin } \\
\text { transporter) }\end{array}$ \\
\hline STXBP1 & 20 & syntaxin binding protein 1 \\
\hline SYN1 & 16 & synapsin I \\
\hline SYNGAP1 & 28 & synaptic Ras GTPase activating protein 1 \\
\hline TCF4 & 19 & transcription factor 4 \\
\hline TSC1 & 15 & Tuberous scleorsis 1 (tuberin stabilizer) \\
\hline TSC2 & 19 & Tuberous sclerosis 2 (tuberin) \\
\hline UBE3A & 19 & $\begin{array}{l}\text { ubiquitin protein ligase E3A (ubiquitin protein } \\
\text { degradation) }\end{array}$ \\
\hline Table & a & autism including syndromic \\
\hline
\end{tabular}

Table 1: Risk genes for autism including syndromic and nonsyndromic cases, linked to at least 15 references in SFARI data base, https://sfari.org/ accessed July 9th 2016

\section{Gene Networks in autism}

Clearly it is of great importance to determine whether genes implicated in autism converge on specific cellular pathways or molecular processes or on specific developmental processes.

A number of large-scale studies on genetic variants in autism have led to the identification of networks of gene involved in autism. It is interesting to note that the networks identified in different studies overlap but are not necessarily identical. Most studies identify genes involved in synaptic functions, genes responsible for chromatin remodeling. Several studies identify genes involved in gene expression and transcription.

Sanders et al. (2015) reported that autism spectrum disorder risk genes could be placed into two major networks ${ }^{7}$. One major network included neuronal elements related to: synapse, neuronal projections, signaling, long-term potentiation, postsynaptic density and cytoskeleton.

The second network included elements related to chromatin organization, nucleosome remodeling complexes, bromodomain 
histone modification complexes, transcription related elements, signaling elements.

Bourgeron (2016) pointed out additional pathways involved in autism ${ }^{8}$. These included genes that encode scaffolding proteins, actin cytoskeleton, and cell adhesion molecules and genes that encode products involved in protein degradation.

\section{Gene expression and Transcription studies}

Mahfouz et al. (2015) emphasized that heterogeneity of the many genes identified as autism candidate genes and the importance of understanding how the autism candidate genes relate to each other in neurodevelopment ${ }^{9}$. To explore this question they utilized the BrainSpan transcriptional atlas of the developing brain (www.brainspan.org) to define the coexpression relationships of 455 autism candidate genes. They noted that there is evidence that genes with similar co-expression patterns likely function together in common cellular pathways.

Mahfouz et al. discovered that the autism candidate genes that showed co-expression dynamics were enriched within transcription modules related to synaptogenesis, mitochondrial function, alternative splicing, protein translation and ubiquitination.

They emphasize that while dysfunctional synaptogenesis may lead to autism, defects in fundamental cellular processes such as protein translation, ubiquitination alternative splicing and mitochondrial function may underlie synaptic dysfunction.

\section{Population studies of autism}

Recently significant information has been garnered that confirms that common variants including inherited variants also play significant roles in autism pathogenesis.

Sandin et al. (2014) reported results of a comprehensive population-based cohort analysis that included 2,049973 Swedish children born 1982 through $2006^{10}$. In this cohort $14,516(0.7 \%)$ were diagnosed with autism spectrum disorder and $5,689(0.2 \%)$ had autistic disorder.

In this study the relative recurrence rate increased with genetic relatedness. Sandin et al. determined that the relative recurrence rate for full siblings was 10.3 , for maternal halfsiblings it was 3.3, and for paternal half-siblings it was 2.9; for cousins it was 2.0. These studies revealed that genetics accounted for $50 \%$ of autism liability.

Gaugler et al. 2014 analyzed data from Swedish families with children born between 1982 and $2007^{11}$. Genotyping was conducted on 3046 samples including 466 cases with autism and 2580 unaffected individuals. They then used the SNP data to determine heritability. Common genetic variants contributed $49 \%$ of the heritability $3 \%$ of heritability was contributed to by rare inherited variants and $3 \%$ of the heritability was contributed to by de novo mutations.

An important conclusion from this study is that currently unaccounted factors contribute approximately $46 \%$ to etiology of autism.

Robinson et al. (2016) analyzed results of genetic studies on large cohorts of autism patients and population based resources that included in total more than 38,000 individuals ${ }^{12}$. Their studies revealed that variants defined as genetic risk factors for autism occur in individuals without a diagnosis of autism and in the general population. They stated that risk factors likely influence social and communication ability.

\section{Conclusions}

Recent studies have revealed that segmental dosage variants, rare de novo nucleotide sequence variants and inherited rare and common sequence variants all play roles in the etiology of autism. Robinson, Neale and Hyman (2015) noted that the complexity of genetic complexity place limits on the value of clinical sequencing in most cases of autism ${ }^{13}$. It is however important to note that sequencing and genomic studies have clinical value in syndromic autism.

Genetic studies have provided insights into the degree of heterogeneity in autism and into the biological pathways underlying autism. Except in specific cases of syndromic autism these studies have however, not yet yielded therapeutic insights.

The recent large population studies have provided important new insights. Of particular importance are studies the have led to the conclusion by Robinson et al. (2016) that a continuum model likely best fits the data and that autism lies at the severe end of the population continuum.

\section{References}

1. DSM-5 The American Psychiatric Association's Diagnostic and Statistical Manual, Fifth Edition (DSM-5) 2013.

2. Smith M, Flodman P, Gargus JJ, et al. Mitochondrial and ion channel gene alterations in autism. Biochim Biophys Acta. 2012 Oct; 1817(10): 1796802

3. Moreno-De-Luca D, Sanders SJ, Willsey AJ, et al. Using large clinical data sets to infer pathogenicity for rare copy number variants in autism cohorts. Mol Psychiatry. 2013 Oct; 18(10): 1090-5.

4. Pinto D, Delaby E, Merico D, et al. Convergence of genes and cellular pathways dysregulated in autism spectrum disorders. Am J Hum Genet. 2014 May 1; 94(5): 677-94.

5. De Rubeis S, He X, Goldberg AP, etal. Synaptic, transcriptional and chromatin genes disrupted in autism. Nature. 2014 Nov 13; 515(7526): 209-15.

6. Chen JA, Peñagarikano O, Belgard TG, et al. The emerging picture of autism spectrum disorder: genetics and pathology. Annu Rev Pathol. Jan 2015; 10: 111-44.

7. SandersSJ.Firstglimpses of theneurobiology of autismspectrum disorder. Curr Opin Genet Dev. 2015 Aug; 33: 80-92.

8. Bourgeron T. Current knowledge on the genetics of autism and propositions for future research. C R Biol. 2016 Jun 8. pii: S16310691(16)30041-5

9. Mahfouz A, Ziats MN, Rennert OM, et al. Shared Pathways Among Autism Candidate Genes Determined by Co-expression Network Analysis of the Developing Human Brain Transcriptome J Mol Neurosci. 2015 Dec; 57(4): 580-94.

10. Sandin S, Lichtenstein P, Kuja-Halkola R, et al. The familial risk of autism. JAMA. 2014 May 7; 311(17): 1770-7.

11. Gaugler T, Klei L, Sanders SJ, et al. Most genetic risk for autism resides with common variation. Nat Genet. 2014 Aug; 46(8): 881-5.

12. Robinson EB, St Pourcain B, Anttila V, et al. Genetic risk for autism spectrum disorders and neuropsychiatric variation in the general population. Nat Genet. 2016 May; 48(5): 552-5. doi: 10.1038/ng.3529. PMID:26998691.

13. Robinson EB, Neale BM, Hyman SE. Genetic research in autism spectrum disorders. Curr Opin Pediatr. 2015 Dec; 27(6): 685-91. doi: 10.1097/ MOP.0000000000000278. PMID:26371945. 